\title{
A Remark Concerning the Charge Operator in Quantum Electrodynamics
}

\author{
Helmut ReEH \\ Max-Planck-Institut für Physik und Astrophysik, München
}

Received June 25, 1969

\begin{abstract}
The convergence of the integral over the local charge density toward the global charge is investigated within the framework of quantum electrodynamics.
\end{abstract}

\section{Introduction}

In relativistic quantum field theories one frequently considers operators which are formal integrals over the entire three dimensional space of the zero component of a conserved quantity. In particular, one writes e.g. for the charge formally

$$
Q=\int j_{0}(x) d^{3} \boldsymbol{x}, \quad \partial^{v} j_{v}(x)=0 .
$$

Recently one has learned that in case the theory does not contain states of arbitrarily small energy-momentum above the vacuum state this expression is to be understood in the sense

$$
\begin{gathered}
(\psi \mid Q \varphi)=\lim _{r \rightarrow \infty}\left(\psi \mid Q_{r} \varphi\right), \\
Q_{r}=\int j_{0}(x) f_{r}(\boldsymbol{x}) \alpha\left(x^{0}\right) d^{3} \boldsymbol{x}, \\
f_{r}(\boldsymbol{x})=f_{0}\left(\frac{\boldsymbol{x}}{r}\right), \quad r \geqq 1, \\
f_{0}(\boldsymbol{x})= \begin{cases}1, & |\boldsymbol{x}| \leqq 1, \\
0, & |\boldsymbol{x}| \geqq 2,\end{cases} \\
\int \alpha\left(x^{0}\right) d x^{0}=1
\end{gathered}
$$

with $f_{0}(\boldsymbol{x}) \in \mathscr{D}\left(\boldsymbol{R}^{3}\right), \alpha\left(x^{0}\right) \in \mathscr{D}\left(\boldsymbol{R}^{1}\right)$. (The notation is explained at the end of this introduction.) $\psi$ and $\varphi$ are not arbitrary vectors in the Hilbert space but are generated from the vacuum state by arbitrary local operators [1-4]. This is from the mathematical point of view a rather weak kind of convergence. Strong or weak convergence in the usual sense cannot occur as is shown e.g. in [3] and [4]. On the other hand the result seems to be rather reasonable from the point of view of physics. 
The question then arises of whether a similar statement holds in case the theory contains states of arbitrarily small energy-momentum and one may suggest that the same result holds provided one does not have the case of a spontaneously broken symmetry [2,4]. It is the intention of the present note to show that this indeed is true for the electric charge in quantum electrodynamics.

\section{Mathematical Notations}

$\boldsymbol{R}^{n}: \quad$ with $n=1,3,4: n$ dimensional real Euclidean space.

$\boldsymbol{R}_{+}^{1}$ : $\quad$ positive real axis including the origin.

$\mathscr{D}\left(\boldsymbol{R}^{n}\right)$ : $\quad$ test function space of arbitrarily often differentiable complex functions over $\boldsymbol{R}^{n}$ with compact support.

$\mathscr{D}(K)$ : $\quad$ subspace of $\mathscr{D}\left(\boldsymbol{R}^{n}\right)$ of functions with support in a subset $K \subset \boldsymbol{R}^{n}$.

$\mathscr{D}\left(\boldsymbol{R}^{4}-0\right)$ : Subspace of $\mathscr{D}\left(\boldsymbol{R}^{4}\right)$ of functions the support of which does not contain the origin.

$\mathscr{S}\left(\boldsymbol{R}^{n}\right)$ : test function space of arbitrarily often differentiable complex functions on $\boldsymbol{R}^{n}$ which as well as all their derivatives vanish faster than any power for increasing arguments.

$\mathscr{D}^{\prime}\left(\boldsymbol{R}^{n}\right), \mathscr{D}^{\prime}(K), \mathscr{D}^{\prime}\left(\boldsymbol{R}^{4}-0\right), \mathscr{S}^{\prime}\left(\boldsymbol{R}^{n}\right)$ : the corresponding spaces of distributions.

$x=\left(x^{0}, \boldsymbol{x}\right)=\left(x^{0}, x^{1}, x^{2}, x^{3}\right)$ : four vectors in $\boldsymbol{R}^{4}$.

$x^{2}=x^{02}-x^{2}$

$g_{v v^{\prime}}: \quad g_{00}=1, g_{i i}=-1$ for $i=1,2,3, g_{v v^{\prime}}=0$ for $v \neq v^{\prime}$.

$L^{1}(d \mu): \quad$ space of $\mu$-Lebesgue integrable complex functions.

$\Phi$ : in front of a term occurring in an equation this symbol means that the equation becomes true if that term is multiplied with a certain finite and non zero constant number.

\section{Assumptions and Statement}

2.1. In quantum electrodynamics we have for the photon field $A_{v}(x)(v=0,1,2,3)$ and the electric current $j_{v}(x)$ the equations

$$
\begin{gathered}
\square A_{v}(x)=j_{v}(x), \\
\partial^{v} j_{v}(x)=0 .
\end{gathered}
$$

We assume that $A_{v}(x)$ is an operator valued tempered distribution transforming like a vector under a unitary representation of the inhomogeneous Lorentz group on the representation space $G$. Unitary is ment with respect to the indefinite metric $(\cdot \mid \cdot)_{G}$ on $G(G$ stands for Gupta) which 
may be expressed by a metric operator $\eta$ and a scalar product $(\cdot \mid \cdot)$ for which $G$ is a Hilbert space

$$
(\psi \mid \chi)_{G}=(\psi \mid \eta \chi), \quad \eta^{+}=\eta, \quad \eta^{2}=1 .
$$

$\eta$ commutes with the four translations $P_{\mu}$. (However, it does not commute with the Lorentz transformations.) Hence the translations are unitarily represented on $G$ which respect to its Hilbert space metric and give rise to a decomposition of unity.

$G$ is assumed to contain a subspace $\mathscr{H}_{1}$ spanned by the states on which the auxiliary condition holds. $\mathscr{H}_{1}$ contains the unique Lorentz invariant vacuum state $\Omega, \mathscr{H}_{1}$ is invariant under Lorentz transformations as well as under all gauge invariant operators (but there are, of course, also non gauge invariant operators leaving $\mathscr{H}_{1}$ invariant). On $\mathscr{H}_{1}$ we have

$$
(\psi \mid \psi)_{G} \geqq 0 .
$$

If one denotes by $\mathscr{H}_{0}$ the set of vectors in $\mathscr{H}_{1}$ with

$$
(\psi \mid \psi)_{G}=0
$$

then the quotient space

$$
\mathscr{H}=\mathscr{H}_{1} / \mathscr{H}_{0}
$$

is the Hilbert space of physical states. On $\mathscr{H}$ we have a unitary representation of the inhomogeneous Lorentz group, the spectrum of the translations vanishes outside the forward light cone.

In addition to the photon field there is the electron field $\psi(x)$. The fields are assumed as local, i.e. they commute resp. anticommute when smeared with test functions the supports of which are space-like to each other. When smeared with test functions, the smeared fields and all polynomials of them are assumed to be applicable to $\Omega$ thus generating a dense set in $G$.

All these assumptions seem to be true for quantum electrodynamics. However, up to now they are proved only for the case of free fields [5].

2.2. Consider now the $Q_{r}$. They are supposed to generate a one parameter group of internal symmetry transformations $\Phi \rightarrow \Phi_{\tau}$ for the local algebra $\mathscr{R}$ formed by the field operators smeared with test functions $\in \mathscr{D}\left(\boldsymbol{R}^{4}\right)$ [6]. In case of a conserved symmetry it holds on $\mathscr{H}$ that the vacuum expectation values are invariant

$$
(\hat{\Omega} \mid \hat{\Phi} \hat{\Omega})=\left(\hat{\Omega} \mid \hat{\Phi}_{\tau} \hat{\Omega}\right)
$$

for all local operators $\hat{\Phi}$ on $\mathscr{H}$. (The elements of $\mathscr{H}$ as well as the induced operators on $\mathscr{H}$ carry a hat in order to distinguish them from those of $\mathscr{H}_{1}$ and $G$.) Hence there exists a uniquely determined family of unitary 
operators

$$
\begin{aligned}
\hat{U}(\tau) & =e^{i \hat{Q} \tau}, \\
\hat{U}(\tau) \hat{\Omega} & =\hat{\Omega}, \quad \hat{Q} \hat{\Omega}=0, \\
\hat{\Phi}_{\tau} & =\hat{U}(\tau) \Phi \hat{U}^{-1}(\tau) .
\end{aligned}
$$

The condition on the $\hat{Q}_{r}$ for generating a conserved symmetry is

$$
\lim _{r \rightarrow \infty}\left(\hat{\Omega} \mid\left[\hat{Q}_{r}, \hat{\Phi}\right] \hat{\Omega}\right)=0
$$

for all local operators on $\mathscr{H}[7,1-4]$. The connection between $Q$ and $Q_{r}$ then is given by $[1,4]$

$$
\begin{aligned}
\lim _{r \rightarrow \infty}\left(\hat{\Omega} \mid \hat{\Phi}_{1} \hat{Q}_{r} \hat{\Phi}_{2} \hat{\Omega}\right) & =\lim _{r \rightarrow \infty}\left(\hat{\Omega} \mid \hat{\Phi}_{1}\left[\hat{Q}_{r}, \hat{\Phi}_{2}\right] \hat{\Omega}\right)+\lim _{r \rightarrow \infty}\left(\hat{\Omega} \mid \hat{\Phi}_{1} \hat{\Phi}_{2} \hat{Q}_{r} \hat{\Omega}\right) \\
& =\left(\hat{\Omega} \mid \hat{\Phi}_{1} \hat{Q} \hat{\Phi}_{2} \hat{\Omega}\right)+\lim _{r \rightarrow \infty}\left(\hat{\Omega} \mid \hat{\Phi}_{1} \hat{\Phi}_{2} \hat{Q}_{r} \hat{\Omega}\right)
\end{aligned}
$$

for all local operators on $\mathscr{H}$. Hence we have to show

$$
\lim _{r \rightarrow \infty}\left(\hat{\Phi} \hat{\Omega} \mid \hat{Q}_{r} \hat{\Omega}\right)=\lim _{r \rightarrow \infty}\left(\Phi \Omega \mid Q_{r} \Omega\right)_{G}=0
$$

for all local operators $\hat{\Phi}$ on $\mathscr{H}$ resp. for all $\Phi \in \mathscr{R}_{1}$ when $\mathscr{R}_{1}$ denotes the operators from $\mathscr{R}$ which leave $\mathscr{H}_{1}$ invariant. This proves at the same time that $\Phi \rightarrow \Phi_{\tau}$ is a conserved symmetry as well as the connection between $Q$ and $Q_{r}$.

Theorem. Under the assumptions mentioned in 2.1 it follows for every $\Phi \in \mathscr{R}_{1}$

$$
\lim _{r \rightarrow \infty}\left(\Phi \Omega \mid Q_{r} \Omega\right)_{G}=0
$$

\section{Proof of the Statement}

We apply an idea of Ref. [8] and [2] and make use of a Jost-LehmannDyson representation derived in [9]. Before doing so, we need some preparations. In particular we extract from (5) together with the assumptions in 2.1 some information concerning the behaviour of the Fourier transform of

$$
\left(\Phi \Omega \mid j_{0}(x) \Omega\right)_{G}
$$

near the origin. Together with the spectrum condition and the relative locality of $j_{0}(x)$ and $\Phi$ this will enable us to prove the statement.

3.1. The assumptions imply a Källen-Lehmann representation

$$
\left(A_{v}(x) \Omega \mid A_{v^{\prime}}(y) \Omega\right)_{G}=\int_{p \in \mathbf{R}^{4}} e^{i p(x-y)}\left\{g_{v v^{\prime}} d \mu_{1}(p)+p_{v} p_{v^{\prime}} d \mu_{2}(p)\right\}
$$

where $d \mu_{2}(p)$ and $d \mu_{1}(p)$ define tempered Lorentzinvariant measures on $\boldsymbol{R}^{4}$. 
[Proof. As usual one concludes that the Fourier transform of (8) is a distribution of the form

$$
g_{\mu \nu} \varrho_{1}(p)+p_{\mu} p_{\nu} \varrho_{2}(p)
$$

where $\varrho_{1}$ and $\varrho_{2}$ are Lorentz invariant. We take that for granted and concentrate on showing that $\varrho_{2}(p) d^{4} p$ defines a measure on $\boldsymbol{R}^{4}$. The commutativity of $\eta$ with $P_{\mu}$ implies that (9) is a measure on $\boldsymbol{R}^{4}$. In particular that is the case for $p_{1} p_{2} \varrho_{2}(p)$. Hence $\varrho_{2}$ is a measure on $\boldsymbol{R}^{4}-0$. The Lorentz invariance of $\varrho_{2}$ will enable us to infer that $\varrho_{2}(p)$ is a measure on $\boldsymbol{R}^{4}$ : $\varrho_{2}(p)$ is clearly in $\mathscr{D}^{\prime}\left(\boldsymbol{R}^{4}-0\right)$. Hence, by a result on Lorentz invariant distributions [10] one has for $f \in \mathscr{D}\left(\boldsymbol{R}^{4}-0\right)$

$$
\int f(p) p_{1} p_{2} \varrho_{2}(p) d^{4} p=\bar{\varrho}_{e}\left[\bar{f}^{e}\right]+\bar{\varrho}_{0}\left[\bar{f}^{0}\right]
$$

with the uniquely determined distributions $\bar{\varrho}_{e} \in \mathscr{D}^{\prime}\left(\boldsymbol{R}^{1}\right), \bar{\varrho}_{0} \in \mathscr{D}^{\prime}\left(\boldsymbol{R}_{+}^{1}\right)$ and

$$
\begin{aligned}
& \bar{f}^{e}(s)=\int p_{1} p_{2} f(p) \delta\left(p^{2}-s\right) d^{4} p, \\
& \bar{f}^{0}(s)=\int p_{1} p_{2} f(p) \varepsilon\left(p_{0}\right) \delta\left(p^{2}-s\right) d^{4} p .
\end{aligned}
$$

Consider now $g(s) \in \mathscr{D}(K)$ with a compact $K \in \boldsymbol{R}^{1}$ and put

$$
\begin{aligned}
& f_{1}(p)=g\left(p^{02}-\boldsymbol{p}^{2}\right) p_{0} p_{1} p_{2} F\left(\boldsymbol{p}^{2}\right), \\
& f_{2}(p)=g\left(p^{02}-\boldsymbol{p}^{2}\right) p_{0}^{2} p_{1} p_{2} F\left(\boldsymbol{p}^{2}\right) .
\end{aligned}
$$

With a suitably chosen non negative $F\left(\boldsymbol{p}^{2}\right) \in \mathscr{D}\left(\boldsymbol{R}^{1}\right)$ we have $f_{1}, f_{2} \in \mathscr{D}\left(\boldsymbol{R}^{4}-0\right)$ and

$$
\begin{aligned}
\bar{f}_{0}^{0}(s) & =g(s) \cdot a, \\
a & =\int p_{1}^{2} p_{2}^{2} F\left(\boldsymbol{p}^{2}\right) d^{3} \boldsymbol{p}>0, \\
\bar{f}_{2}^{e}(s) & =g(s) h(s), \\
h(s) & =\int \sqrt{|\boldsymbol{p}|^{2}+s} p_{1}^{2} p_{2}^{2} F\left(\boldsymbol{p}^{2}\right) d^{3} \boldsymbol{p} .
\end{aligned}
$$

$h(s)$ is apparently infinitely often differentiable and unequal zero for all $s \geqq 0$. By choosing $F\left(p^{2}\right)$ appropriately, this stays true for all values $s \in K$. We now replace $f_{1}$ and $f_{2}$ by respectively

$$
\begin{aligned}
& f_{3}(p)=\frac{1}{a} f_{1}(p) \\
& f_{4}(p)=\left\{\begin{array}{lll}
\frac{g\left(p^{0^{2}}-\boldsymbol{p}^{2}\right)}{h\left(p^{0^{2}}-\boldsymbol{p}^{2}\right)} p_{0}^{2} p_{1} p_{2} F\left(\boldsymbol{p}^{2}\right) & \text { for } & p \in \operatorname{supp} f_{2} \\
0 & \text { for } & p \notin \operatorname{supp} f_{2},
\end{array}\right.
\end{aligned}
$$

23 Commun. math. Phys., Vol 14 
which are still in $\mathscr{D}\left(\boldsymbol{R}^{4}-0\right)$. Then we have

$$
\begin{aligned}
& \bar{f}_{3}^{e}(s)=0, \\
& \bar{f}_{3}^{0}(s)=g(s), \\
& \bar{f}_{4}^{e}(s)=g(s), \\
& \bar{f}_{4}^{0}(s)=0,
\end{aligned}
$$

and furthermore

$$
\begin{gathered}
\sup _{p \in \mathbf{R}^{4}} f_{3}(p) p_{1} p_{2} \leqq \sup _{s \in K} g(s) C_{K}, \\
C^{K}=\sup _{p^{02}-\boldsymbol{p}^{2} \in K} a \cdot p_{0} p_{1}^{2} p_{2}^{2} F\left(\boldsymbol{p}^{2}\right), \\
\sup _{p \in \mathbf{R}^{4}} f_{4}(p) p_{1} p_{2} \leqq \sup _{s \in K} g(s) C_{K}^{\prime}, \\
C_{\boldsymbol{K}^{\prime}}=\sup _{p^{0^{2}}-\boldsymbol{p}^{2} \in K} \frac{p_{0}^{2} p_{1}^{2} p_{2}^{2}}{h\left(p_{0}^{2}-p^{2}\right)} F\left(\boldsymbol{p}^{2}\right) .
\end{gathered}
$$

Now we know that $\varrho_{2}(p)$ is a measure, i.e. a distribution of order zero on $\mathscr{D}\left(\boldsymbol{R}^{4}-0\right)$. Hence

$$
\begin{aligned}
\left|\varrho_{e}[g]\right|=\left|\int f_{4}(p) p_{1} p_{2} \varrho_{2}(p) d^{4} p\right| & \leqq \sup _{p \in \mathbf{R}^{4}} f_{4}(p) p_{1} p_{2} C_{1} \\
& \leqq \sup _{s \in K} g(s) C_{1} \cdot C_{K}^{\prime}
\end{aligned}
$$

and

$$
\begin{aligned}
\left|\varrho_{0}[g]\right|=\left|\int f_{3}(p) p_{1} p_{2} \varrho_{2}(p) d^{4} p\right| & \leqq \sup _{3}(p) p_{1} p_{2} C_{2} \\
& \leqq \sup _{s \in K} g(s) C_{2} \cdot C_{K}
\end{aligned}
$$

$\left(C_{1}\right.$ and $C_{2}$ depend on $K$ too).

Hence [11] $\bar{\varrho}_{e}$ and $\bar{\varrho}_{0}$ are Radon measures on $\boldsymbol{R}^{1}$ and $\boldsymbol{R}_{+}^{1}$ respectively.

We know that $p_{1} p_{2} \varrho_{2}(p)$ is a measure on $\boldsymbol{R}^{4}$ with value zero at the origin. Hence it is fixed uniquely by its values on testfunctions from $\mathscr{D}\left(\boldsymbol{R}^{4}-0\right)$ and we have for $f \in \mathscr{D}\left(\boldsymbol{R}^{4}\right)$

$$
\int f(p) p_{1} p_{2} \varrho_{2}(p) d^{4} p=\bar{\varrho}_{e}\left[\bar{f}^{e}\right]+\bar{\varrho}_{0}\left[\bar{f}^{0}\right] .
$$

We extend now $\varrho_{2}(p)$ to all of $\mathscr{D}\left(\boldsymbol{R}^{4}\right)$ by

with

$$
\int f(p) \varrho_{2}(p) d^{4} p=\bar{\varrho}_{e}\left[\overline{\bar{f}}^{e}\right]+\bar{\varrho}_{0}\left[\overline{\bar{f}}^{0}\right]
$$

$$
\begin{aligned}
& \overline{\bar{f}}^{e}(s)=\int f(p) \delta\left(p^{2}-s\right) d^{4} p, \\
& {\overline{\bar{f}^{0}}}^{0}(s)=\int f(p) \varepsilon\left(p^{0}\right) \delta\left(p^{2}-s\right) d^{4} p
\end{aligned}
$$

which is possible, since $\overline{\bar{f}}^{e}(s), \overline{\bar{f}}^{0}(s)$ are continuous on $\boldsymbol{R}^{1}$ and $\boldsymbol{R}_{+}^{1}$ respectively. Furthermore we have for $f \in \mathscr{D}\left(K^{1}\right)$ with a compact $K^{1} \subset \boldsymbol{R}^{4}$ 
and with suitable numbers $C_{K^{1}}^{1}, C_{K^{1}}^{2}$

$$
\begin{aligned}
& \sup _{s \in \mathbf{R}^{1}} \overline{\bar{f}}^{e}(s) \leqq C_{\boldsymbol{K}^{1}}^{1} \sup _{p \in \boldsymbol{K}^{1}} f(p), \\
& \sup _{s \in \boldsymbol{R}^{1}} \overline{\bar{f}}^{0}(s) \leqq C_{\boldsymbol{K}^{1}}^{2} \sup _{p \in K^{1}} f(p) .
\end{aligned}
$$

Hence $\varrho_{2}(p) d p \equiv d \mu_{2}(p)$ defines a Radon measure on $\boldsymbol{R}^{4}$, and so does $p_{v} p_{v^{\prime}} \varrho_{2} d p=p_{v} p_{v^{\prime}} d \mu_{2}(p)$ and $\varrho_{1}(p) d p \equiv d \mu_{1}(p)$. The temperedness is implied by the temperedness of $\left.A_{v}(x)\right]$.

From (5) and (6) it follows that

$$
\left(j_{v}(x) \Omega \mid j_{v^{\prime}}(y) \Omega\right)_{G}=\int_{p \in \mathbf{R}^{4}} e^{i p(x-y)}\left(p^{2}\right)^{2}\left(p_{v} p_{v^{\prime}}-p^{2} g_{v v^{\prime}}\right) d \mu_{2}(p)
$$

and in particular

$$
\left(j_{0}(x) \Omega \mid j_{0}(y) \Omega\right)_{G}=\int e^{i p(x-y)}\left(p^{2}\right)^{2}|\boldsymbol{p}|^{2} d \mu_{2}(p) .
$$

Since the current is gauge invariant, it follows that $\mu_{2}(\mathrm{p})$ is a positive measure off the light cone. The spectrum condition on $\mathscr{H}$ implies that $p^{2} d \mu_{2}(p)$ vanishes outside the forward light cone.

3.2. Consider $\Phi \in \mathscr{R}_{1}$, define $\Phi(x)=U(x) \Phi U^{-1}(x)$ where

$$
U(x)=\int e^{-i p x} d E(p)
$$

is the unitary representation of the space-time translations, $x \in \boldsymbol{R}^{4}$, $p \in \boldsymbol{R}^{4}, p x=p^{0} x^{0}-\boldsymbol{x} \boldsymbol{p}$. In

$$
\left(\Phi \Omega \mid j_{0}(x) \Omega\right)_{G}=\int e^{-i p x} d \sigma(p) .
$$

$\sigma(p)$ is a tempered complex measure on $\boldsymbol{R}^{4}$ which is locally finite. (This follows like the next equations immediately from the translation invariance and from $j_{0}(x)$ being an operator valued tempered distribution.) $\sigma(p)$ vanishes outside the forward light cone due to the spectrum condition. Hence $\tilde{\alpha}\left(p^{0}\right) d \sigma(p)$ with $\tilde{\alpha}\left(p^{0}\right) \in \mathscr{S}\left(\boldsymbol{R}^{1}\right)$ is a finite complex measure on $\boldsymbol{R}^{4}$. With the notation $\Phi[g]=\int \Phi(x) g(x) d^{4} x$, etc., we have due to translational invariance $(\check{g}(x) \equiv g(-x))$ :

$$
\begin{aligned}
\left(\Phi[g] \Omega \mid j_{0}(x) \Omega\right)_{G} & =\left(\Phi(-x) \Omega \mid j_{0}[\check{g}] \Omega\right)_{G} \\
& =\int e^{-i p x}\left(\Phi \Omega \mid \eta d E(p) j_{0}[\check{g}] \Omega\right) \\
& =\int e^{-i p x} \tilde{g}(p) d \sigma(p),
\end{aligned}
$$

with $\tilde{g}(p)=\int e^{i p y} g(y) d^{4} y$. This holds for all test functions $g \in \mathscr{S}\left(\boldsymbol{R}^{4}\right)$ and all $x \in \boldsymbol{R}^{4}$. Therefore, it follows for every Borel set $\Delta \in \boldsymbol{R}^{4}$ that

$$
\int_{p \in \Delta} \tilde{g}(p) d \sigma(p)=\int_{p \in \Delta}\left(\Phi \Omega \mid d E(p) j_{0}[\check{g}] \Omega\right)_{G} .
$$


From this we get by Schwarz' inequality on $\mathscr{H}_{1}$

$$
\begin{aligned}
\left|\int_{\Delta} \tilde{g}(p) d \sigma(p)\right| & \leqq\|\Phi \Omega\|_{G}\left\{\int_{\Delta}\left(j_{0}[\check{g}] \Omega \mid \mathrm{d} E(p) j_{0}[\check{g}] \Omega\right)_{G}\right\}^{1 / 2} \\
& =\|\Phi \Omega\|_{G}\left\{\int_{\Delta}|\tilde{g}(p)|^{2}\left(p^{2}\right)^{2}|\boldsymbol{p}|^{2} d \mu_{2}(p)\right\}^{1 / 2} .
\end{aligned}
$$

We now put $\tilde{g}(p)=\tilde{g}_{1}(p) \tilde{\alpha}\left(p^{0}\right)$ with $\tilde{\alpha}\left(p^{0}\right) \in \mathscr{S}\left(\boldsymbol{R}^{1}\right), \tilde{g}_{1}(p) \in \mathscr{S}\left(\boldsymbol{R}^{4}\right)$ and write $\tilde{g}(p)$ instead of $\tilde{g}_{1}(p)$

$$
\left|\int_{\Delta} \tilde{g}(p) \tilde{\alpha}\left(p^{0}\right) d \sigma(p)\right| \leqq\|\Phi \Omega\|_{G}\left\{\int_{\Delta}|\tilde{g}(p)|^{2}\left(p^{2}\right)^{2}|\boldsymbol{p}|^{2}\left|\tilde{\alpha}\left(p^{0}\right)\right|^{2} d \mu_{2}(p)\right\}^{1 / 2} .
$$

$\tilde{\alpha}\left(p^{0}\right) d \sigma(p)$ and $\left|\tilde{\alpha}\left(p^{0}\right)\right|^{2} d \mu_{2}(p)$ define finite measures on $\boldsymbol{R}^{4}$. (11) holds for every continuous function $\tilde{g}(p)$ bounded by a polynomial for large $p$, in particular for $\tilde{g}(p)=1$. Application of the Radon-Nikodym theorem [12] implies

$$
\tilde{\alpha}\left(p^{0}\right) d \sigma(p)=m(p)\left(p^{2}\right)^{2}|\boldsymbol{p}|^{2}\left|\tilde{\alpha}\left(p^{0}\right)\right|^{2} d \mu_{2}(p)
$$

with $m(p) \in L^{1}\left(\left(p^{2}\right)|\boldsymbol{p}|^{2}\left|\tilde{\alpha}\left(p^{0}\right)\right|^{2} d \mu_{2}(p)\right)$. Inserting this into (11), we get $\left.\left|\int_{\Delta} \tilde{g}(p) m(p)\left(p^{2}\right)^{2}\right| \boldsymbol{p}\right|^{2}\left|\tilde{\alpha}\left(p^{0}\right)\right|^{2} d \mu_{2}(p) \mid$

$$
\leqq\|\Phi \Omega\|_{G}\left\{\int_{\Delta}|\tilde{g}(p)|^{2}\left(p^{2}\right)^{2}|\boldsymbol{p}|^{2}\left|\tilde{\alpha}\left(p^{0}\right)\right|^{2} d \mu_{2}(p)\right\}^{1 / 2} .
$$

Let now $\Delta$ be bounded, contain the origin and let $\tilde{g}(p) \geqq 0$ on $\Delta$. If $m(p)$ is decomposed into its real and imaginary parts, then (12) holds for each part separately. By decomposing $\Delta$ into $\Delta_{r}^{+} \cup \Delta_{r}^{-}$(or $\Delta_{i}^{+} \cup \Delta_{i}^{-}$ respectively) with $\operatorname{Re} m(p) \geqq 0$ on $\Delta_{r}^{+}, \operatorname{Re} m(p)<0$ on $\Delta_{r}^{-}$(respectively for $\operatorname{Im} m(p))[14]$, and by applying (12) on $\Delta_{r}^{+}$and $\Delta_{r}^{-}\left(\right.$or $\Delta_{i}^{+}, \Delta_{i}^{-}$) separately, one shows that

$$
\begin{aligned}
\int_{\Delta} \tilde{g}(p)|m(p)|\left(p^{2}\right)^{2}|\boldsymbol{p}|^{2} & \left|\tilde{\alpha}\left(p^{0}\right)\right|^{2} d \mu_{2}(p) \\
& \leqq 2 \sqrt{2}\|\Phi \Omega\|_{G}\left\{\int_{\Delta}|\tilde{g}(p)|^{2}\left(p^{2}\right)^{2}|\boldsymbol{p}|^{2}\left|\tilde{\alpha}\left(p^{0}\right)\right|^{2} d \mu_{2}(p)\right\}^{1 / 2} .
\end{aligned}
$$

We let now $\tilde{g}(p)$ converge toward $\left(p^{2}|\boldsymbol{p}|\right)^{-1}$ pointwise on $\Delta$. Fatou's lemma [13] shows that

$$
m(p) \in L^{1}\left(p^{2}|\boldsymbol{p}|\right)\left|\alpha\left(p^{0}\right)\right|^{2} d \mu_{2}(p) .
$$

Since $\mu_{2}(p)$ is tempered and $p^{2} d \mu_{2}(p)$ vanishes outside the forward light cone, this stays true (compare the last inequality!) if $m(p)$ is multiplied by any power of the components of $p$. 
3.3. Consider now with $\alpha\left(x^{0}\right) \in \mathscr{D}\left(\boldsymbol{R}^{1}\right)$

$$
\left(\Phi[\check{\alpha}] \Omega \mid j_{0}(x) \Omega\right)_{G}
$$

the Fourier transform of which, $\tilde{\alpha}\left(p^{0}\right) d \sigma(p)$, is discussed above. According to [9] one has for this a Jost-Lehmann-Dyson representation $\left(\Phi[\check{\alpha}] \Omega \mid j_{0}(x) \Omega\right)_{G}$

$$
\begin{aligned}
& =\not{\int} \int_{D_{1}} d^{3} \xi^{\prime} \int d \sigma(p) \tilde{\alpha}\left(p_{0}\right) e^{-i \boldsymbol{p} \boldsymbol{\xi}^{\prime}}\left(\frac{\partial}{\partial x^{0}}-i p^{0}\right) \Delta_{\sqrt{\boldsymbol{p}^{2}}}^{+}\left(x-\xi^{\prime}\right) \\
& =\not{C} \int_{D^{\prime}} d^{3} \xi^{\prime} \int d \mu_{2}(p) m(p)\left(p^{2}\right)^{2}|\boldsymbol{p}|^{2}\left|\tilde{\alpha}\left(p^{0}\right)\right|^{2} e^{-i \boldsymbol{p} \xi^{\prime}}\left(\frac{\partial}{\partial x^{0}}-i p^{0}\right) \Delta_{\sqrt{\boldsymbol{p}^{2}}}^{+}\left(x-\xi^{\prime}\right),
\end{aligned}
$$

where $D_{1}$ denotes a compact region in $\boldsymbol{R}^{3}$. We keep now $x^{0}$ finite (in fact, we shall put it zero) and let $\boldsymbol{x}$ increase. Since $D_{1}$ is compact, $x-\boldsymbol{\xi}^{\prime}$ will become space-like for sufficiently large $\boldsymbol{x}$, and we get (observe the factor $p^{2}$ !) $\left(\Phi[\check{\alpha}] \Omega \mid j_{0}(x) \Omega\right)_{G}$

$$
\begin{array}{r}
=\not \int_{D_{1}} d^{3} \xi^{\prime} \int d \mu_{2}(p) m(p) p^{2}|\boldsymbol{p}|^{2}\left|\tilde{\alpha}\left(p^{0}\right)\right|^{2} e^{-i \boldsymbol{p} \xi^{\prime}}\left(\frac{\partial}{\partial x^{0}}-i p^{0}\right) \\
\cdot \sqrt{p^{2}} \frac{K_{1}\left(\sqrt{p^{2}} \sqrt{-(x-\xi)^{2}}\right)}{\sqrt{-(x-\xi)^{2}}}
\end{array}
$$

with the cylindrical function $K_{1}$. Hence for $|\boldsymbol{x}|$ sufficiently large

$$
\begin{aligned}
& \left|\left(\Phi[\check{\alpha}] \Omega \mid j_{0}(x) \Omega\right)_{G}\right| \\
& \leqq 屯\left|\int_{D_{1}} d^{3} \xi^{\prime}\right| \cdot \int|m(p)| p^{2}|\boldsymbol{p}|^{2} p^{0}\left|\tilde{\alpha}\left(p^{0}\right)\right|^{2} d \mu_{2}(p) \cdot \sup _{y \in \boldsymbol{R}^{1}}\left(y^{3} K_{1}(y)\right) \frac{1}{|\boldsymbol{x}|^{4}} \\
& +\nsubseteq\left|\int_{D_{1}} d^{3} \xi\right| \cdot \int|m(p)| p^{2}|\boldsymbol{p}|^{2}\left|\tilde{\alpha}\left(p^{0}\right)\right|^{2} d \mu_{2}(p) \cdot \sup _{y \in \boldsymbol{R}^{1}}\left(y^{3} K_{1}(y)\right) \frac{x^{0}}{|\boldsymbol{x}|^{6}} \\
& +\Phi\left|\int_{D_{1}} d^{3} \xi\right| \cdot \int|m(p)| p^{2}|\boldsymbol{p}|^{2}\left|\tilde{\alpha}\left(p^{0}\right)\right|^{2} d \mu_{2}(p) \cdot \sup _{y \in \boldsymbol{R}^{1}}\left(y^{4} \frac{\partial}{\partial y} K_{1}(y)\right) \frac{x^{0}}{|\boldsymbol{x}|^{6}} \text {. }
\end{aligned}
$$

Since for small $y K_{1}(y) \approx 1 / y$, and since for large $y K_{1}$ drops exponentially, it follows for $x^{0}=0$ and $|\boldsymbol{x}|$ large

$$
\left|\left(\Phi[\check{\alpha}] \Omega \mid j_{0}(x) \Omega\right)_{G}\right| \leqq \nsubseteq \frac{1}{|\boldsymbol{x}|^{4}} .
$$


In particular, we see now that

exists.

$$
\lim _{r \rightarrow \infty}\left(\Phi \Omega \mid Q_{r} \Omega\right)
$$

3.4. We may write

with

$$
\left(\Phi[\check{\alpha}] \Omega \mid j_{0}(x) \Omega\right)_{G}=\sum_{i=1}^{3} \frac{\partial}{\partial x_{i}} F_{i}(x)
$$

$F_{i}(x)=\varnothing \int_{D_{1}} d^{3} \xi^{\prime} \int d \mu_{2}(p) m(p)\left(p^{2}\right)^{2} p_{i}\left|\tilde{\alpha}\left(p^{0}\right)\right|^{2} e^{-i p \xi^{\prime}}\left(\frac{\partial}{\partial x^{0}}-i p^{0}\right) \Delta_{\sqrt{p^{2}}}^{+}\left(x-\xi^{\prime}\right)$.

As in 3.3 it follows for $x^{0}=0$ and $|\boldsymbol{x}|$ large

$$
\left|F_{i}(x)\right| \leqq \nsubseteq \frac{1}{|x|^{4}}
$$

Hence the Fourier transform $\tilde{F}_{i}(p)$ of $F_{i}(x, 0)$ is bounded and continuous in $p$ and

$$
\begin{aligned}
\left(\Phi \Omega \mid Q_{r} \Omega\right)_{G} & =\int \tilde{f_{r}}(\boldsymbol{p}) p_{i} \tilde{F}_{i}(\boldsymbol{p}) d^{3} \boldsymbol{p} \\
& =\frac{1}{r} \int \tilde{f}_{0}(\boldsymbol{q}) q_{i} \tilde{F}_{i}\left(\frac{\boldsymbol{q}_{i}}{r}\right) d^{3} \boldsymbol{q}
\end{aligned}
$$

which converges toward zero for $r \rightarrow \infty$ as it was stated above.

Acknowledgement. I benefitted from discussions with Dr. P. Breitenlohner, Dr. D. Maison, E. Rudolph and Dr. S. Schlieder.

Note added in proof. As it was pointed out to the author by J. A. Swieca, perturbation theory indicates that $\varrho_{2}(p)$ has a contribution of the first derivative of a delta function on the light cone. The preceding proof works also if such a contribution is present because it does not show up in the two point function of the current, and our statement stays true. However, the assumption that $\eta$ commutes with $P_{\mu}$ then has to be modified. - I thank Prof. Swieca for this information.

\section{References}

1. Robinson, D. W.: Istanbul lectures 1966.

2. Swieca, J. A.: Nuovo Cimento 52, 242 (1967).

3. Schroer, B., and P. Stichel: Commun. Math. Phys. 3, 258 (1966).

4. Reeh, H.: Fortschr. Physik 16, 687 (1968).

5. cf. Gårding, L., and A. S. Wightman: Arkiv Fys. 28, 129-184 (1965) and the standard textbooks on quantum electrodynamics.

6. See e.g. [4]. "Internal" means that the space-time support of the operators is not changed. Compare in this connection also: Maison, D.: Symmetry Transformations from local currents, to be published. 
7. Kastler, D., D. W. Robinson, and A. Swieca: Commun. Math. Phys. 2, 108 (1966).

8. Ezawa, H., and J. A. Swieca: Commun. Math. Phys. 5, 330 (1967).

9. Araki, H., K. Hepp, and D. Ruelle: Helv. Phys. Acta 35, 164 (1962).

10. Gårding, L., and J.L.Lions: Nuovo Cimento 14, Suppl. 9 (1959), Sect. 8; Rieckers, A., and W. Güttinger: Commun. Math. Phys. 7, 190 (1967).

11. cf. e.g. Treves, F.: Topological vector spaces, distributions and kernels, Sect. 21. New York-London: Academic Press 1967.

12. cf. e.g. Berberian, S. K.: Measure and integration, Sect. 52, Theorem 1. New York: Macmillan Comp. 1965.

13. cf. e.g. Berberian, S. K.: 1.c. [12], Sect. 32, Theorem 1.

14. Compare e.g. Berberian, S. K.: 1.c. [12], Sect. 49 for the decomposition into positive and negative parts.

\section{H. Reeh}

Max-Planck-Institut für Physik und Astrophysik

8000 München 23, Föhringer Ring 6 